\title{
Aligning Elements of the Identification Process: Implications for Hidden Exceptionalities
}

\author{
Ian A. Matheson and Kyle Robinson \\ Queen's University
}

\begin{abstract}
Students with learning disabilities and other high-incidence hidden exceptionalities (HIHE) can struggle with secondary difficulties including low confidence in their abilities (e.g., Klassen, 2010) along with primary difficulties related to their exceptionality. The purpose of this paper is to highlight the importance of the perspective of individuals with hidden exceptionalities with regard to maladaptive beliefs they can form. Using learning disabilities as a platform for discussing HIHE, we unpack two types of maladaptive beliefs - related to generalizations and mindset-and suggest adaptive alternative beliefs that can promote adaptive behaviour. The focus on maladaptive beliefs draws attention to the identification process, and specifically the types of variables that can influence how well aligned parts of the process are for particular students. We present a framework that contextualizes individual beliefs within the identification process, and what other variables determine alignment of parts within the process. The framework can be used to support educators through the identification process, as well as researchers in providing direction for future research.
\end{abstract}

In addition to the learning difficulties that individuals with learning disabilities (LD) face, they also tend to present with low confidence in their abilities. For example, researchers have found that individuals with LD present with low academic (Baird, Scott, Dearing, \& Hamill, 2009), social (Lackaye, Margalit, Ziv, \& Ziman, 2006), and self-regulatory efficacy (Klassen, 2010) compared to their non-LD peers. While low confidence can develop through a history of learning-related struggles, it may also emerge in part because of a students' identification with, and beliefs about, an assigned label. Although 
there are a number of supportive features that come with formal identification (e.g., funding), how the identification process unfolds and is communicated to the student can impact their beliefs about their abilities.

The purpose of this paper is to emphasize the importance of the perspective of students with "hidden" exceptionalities, using LD as a platform for contextualizing current issues and possible remediations. Without changing structures and processes of identification and support, it is possible to address maladaptive beliefs and perspectives that students with hidden exceptionalities can form about their learning challenges and that are rooted in inaccuracies (generalizations) and self-imposed limitations (maladaptive mindset). When students' maladaptive beliefs promote inaction (learned helplessness) or other behaviours that avoid growth in subject areas, they earn another label_- "at risk," reflecting a general pattern of disengagement from school (DeLuca et al., 2010). Despite educators' efforts to support skill deficits and consequent learning difficulties, the academic outcomes of students with hidden exceptionalities, both short and long term, come down to the learning-related experiences they have. In this paper, we discuss how identifications are made in North America, identify maladaptive beliefs and adaptive alternatives, and highlight the importance of non-academic interventions to supplement interventions focused on academic challenges. As the most common type of exceptionality that is identified (Learning Disabilities Association of Ontario, 2018), we use LD as an entry point to considering the power of students' beliefs about their hidden exceptionalities.

\section{Identification of Learning Disabilities in North America}

The term hidden exceptionalities refers to a group of exceptionalities that are not immediately visible and, in many cases, may be actively concealed by the identified individuals (e.g., avoiding specific tasks or situations) from peers for personal and/or social reasons. These exceptionalities typically include LD, emotional and behavioural exceptionalities, mild intellectual disabilities, and level-1 autism spectrum disorder (American Psychiatric Association [APA], 2013), among others (Gage, Lierhiemer, \& Goran, 2012). Each of the above-mentioned exceptionalities are also considered to be high-incidence, as they are prevalent within Canadian classrooms, and can therefore be referred to as high-incidence hidden exceptionalities (HIHE).

Along with allowing for the provision of funding for identified students, identifying students with an exceptionality can also promote social action to support a recognized exceptionality (e.g., seeking appropriate resources) and can give educators appropriate language to use when talking about ways to support students with recognized learning needs (Gargiulo, 2012). However, past research has shown that identifying students with LD is associated with teachers' lowered expectations of those students, as well as with more negative stereotypes and attitudes about students with LD from their peers without LD (Osterholm, Nash, \& Kritsonis, 2007). Other research has shown an increase in the self-esteem of students with LD following their identification, possibly due to the adoption of a view of LD as being limited to a specific subject area and therefore as manageable, or to a change in students' comparison group from one without LD to one with LD (MacMaster, Donovan, \& MacIntyre, 2002). Whether they are accurate or not, students' 
beliefs about their abilities could logically impact the types of activities they engage in, as well as those they try to avoid when possible. Given the benefits and ongoing practice of formally identifying students with exceptionalities, a focus needs to turn to understanding the perspective and experience of students who receive a formal identification for an exceptionality that could be concealed and therefore considered hidden.

A number of models exist for understanding disability, though medical models that emphasize deficits or impairments as the root cause of problems that an individual may experience have dominated most education systems, including many parts of Canada (Hutchinson, 2017) and the United States (Cortiella \& Horowitz, 2014). These systems of identification use definitions and assessments based in part or in whole on the American Psychiatric Association's Diagnostic and Statistical Manual of Mental Disorders (DSM; APA, 2013). For example, the fifth and most recent edition of the DSM defines LD (referred to as "specific learning difficulties") as "difficulties learning and using academic skills" (DSM-5; APA, 2013, p. 66). These difficulties must be indicated by one of six symptoms: (a) inaccurate or slow, effortful reading; (b) difficulty comprehending; (c) difficulties with spelling; (d) difficulties with written expression; (e) difficulties with number sense, number facts, or calculation; or (f) difficulties with mathematical reading.

Within educational systems that take a medical perspective on exceptionalities like LD, often there is not a need for students to have received a medical diagnosis by a trained physician or other medical professional. In these circumstances, students may receive an identification with an outline of their specific LD and the associated learning challenges, as well as guidelines for how educators may choose to support that impairment.

In Ontario, where the authors are located, teachers can receive information about a student's identified exceptionality from their Individual Education Plan (IEP; called an Individualized Learning Plan [ILP] or Individual Program Plan [IPP] in other parts of the world) - a document that purports to describe the special education program and/or services that a particular student requires based on a comprehensive assessment of the student's needs and strengths - that is, the needs and strengths that affect the student's ability both to learn and to demonstrate their learning (Ontario Ministry of Education [OME], 2017). However, the classification system used to create the IEP is based upon a strict adherence to certain guidelines. Exceptionalities are often grouped into larger categories; in Ontario there are five such categories: behaviour, communication, intellectual, physical, and multiple (Finlay, 2011).

Simeonsson (2009) suggested these kinds of categories are "often idiosyncratic to specific service systems" (p. 70). They do not fully encapsulate the life and experiences of a person, which is necessary to truly understand their needs (Lillvist, 2010; Simeonsson, 2009; Simeonsson, Scarborough, \& Hebbeler, 2006). As Stein (2006) argued, it is important to focus on the functioning needs, not the exceptionality label:

The functioning of children is always a moving target, as children mature at different rates, live in different cultures with different expectations of independence and self-sufficiency, and grow up in environments that vary markedly in the demands that they place on the performance of activities by children. (p. 145)

Lillvist (2010) expanded on this idea, suggesting, "many children diagnosed with the same medical condition show an array of different consequences resulting from the 
condition" (p. 1131). While students with LD may present with similar learning difficulties, their experience of these difficulties could vary greatly. In the DSM-5 (APA, 2013), three different types of LD are identified based on problems that students face in specific subject areas. Students may be identified with a LD in reading, writing, or mathematics, or some combination of the three. However, even within each type of LD, there is variation among students in how they experience their LD (Geary, 2006), and some researchers suggest that further subtypes may be required within each type of LD in order to recognize the variation within each type (Buttner \& Hasselhorn, 2011).

\section{Maladaptive Beliefs}

During the first of many tutoring sessions, a literacy tutor and student sit beside each other with several passages of text laid out in front of them on the table. The tutor selects a passage for the student to read-a page consisting of one large diagram of an eco-friendly fish farm and seven captions throughout the diagram to explain how the farm functions. Before the student attempts to begin reading, they look at the tutor and say, "You know, I won't be able to do this-I have a learning disability."

In the example above-based on the first author's experiences tutoring - the student's response to the proposed task gives us a glimpse into the types of beliefs students with LD may possess related to their learning. While students with LD may possess one of a multitude of perspectives about what their exceptionality means, the above example represents what could be considered a maladaptive response reflecting maladaptive beliefs. In what follows, we will unpack the example to identify two distinct but related maladaptive beliefs and explain how they could be considered maladaptive responses to learning experiences.

\section{Generalizations}

In the example above, it appears that the student believes that they will be unable to engage successfully in a reading task simply because of LD-a generalization likely based on past struggles with reading tasks.

Individuals with LD may make generalizations about their exceptionality that may be due in part to the language educators use in discussing LD; a generalization is the construction of a general concept from a specific case. Specific deficits or difficulties may not be expressed in language that the students experiencing them understand, and students' generalizations about the nature of their LD then become an issue of access to information about LD due to its being too abstract or complex. Further, educators' actions to support students may also be influenced by the language used to describe LD. In a recent study, teachers had more confidence in their abilities to support students with "reading difficulties" than students with "dyslexia," viewing the latter as more of a fixed condition than the former (Gibbs \& Elliott, 2015). Given that they are often the primary source of support for students with LD in the classroom, the beliefs that teachers have about students' abilities may influence students' own beliefs about abilities (Mouzourou, 2009). Both educators and students would be less likely to make generalizations about the challenges LD present to individual students when the nature of specific difficulties or deficits are communicated clearly to them. 
Regarding the recognized types of LD in reading, writing, and mathematics (APA, 2013), each type reflects a subject area that is multi-faceted. Within reading LD, students may struggle with word-level difficulties, problems with fluency, or challenges with comprehension. Within writing LD, students may experience difficulty with fine-motor skills, spelling, or composition of passages. Within mathematics LD, students may struggle with numerical operations, computation speed, or problem solving. These lists are partial, but represent the multi-dimensional nature of each subject, and therefore, the myriad difficulties students can experience even within a single subject.

Students who struggle in one particular area within a subject may overgeneralize this difficulty to all dimensions of the subject and believe that they are not competent within that subject area. While some students with LD may experience difficulties in one component of a multi-dimensional skill (e.g., reading comprehension), they might adopt the belief that their exceptionality reflects a general difficulty with the multi-dimensional skill (e.g., reading), which can be considered a generalization. At best, these students would be able to communicate that they have learning difficulties with a general multidimensional skillset with no awareness of their specific learning needs within the skillset. These generalizations could be considered overgeneralizations, as they involve the belief that one has more specific difficulties than one actually does.

While overgeneralizations can be limiting for a student, undergeneralizations of difficulties can also be maladaptive. Some research supports the idea that LDs reflect underlying cognitive deficits that manifest in a number of ways related to specific academic skills (Buttner \& Hasselhorn, 2011). Executive functions refer to cognitive abilities or actions underlying the higher order thinking processes that are necessary for effective reading, writing, and mathematics (Kane \& Engle, 2002). Students may be identified with LD as a result of an identified deficit in an executive function that is paramount to learning. For example, fluency in reading and computation speed in mathematics may share a common cognitive property - the ability to shift between sources of information. Shifting is considered as an executive function in many models (e.g., Miyake et al., 2000; Naglieri \& Goldstein, 2013). A deficit in fluency should affect learning in more than one subject; and yet a student may only be identified as having a learning disability in reading, as the deficit could manifest most obviously in that multidimensional skillset. Consequently, difficulties experienced in other subjects as a result of the same skill deficit may be viewed as indications of a lack of ability in those subjects.

With the text described in the vignette earlier in this paper, a student with a planning deficit would likely struggle to determine a place to begin reading when there is no obvious starting point, as well as in determining the order in which to proceed through the text. Similarly, a student with a deficit in planning might experience difficulties solving complex multi-step problems in math, or with composition of a story or essay in writing. For students with LD who believe their difficulty is limited to one subject area, such as reading comprehension, it may be confusing and frustrating when difficulties also occur in math problem solving or in writing composition. Without understanding the nature of their specific cognitive deficit, students with LD could lose interest and reduce their effort over time in school subjects due to on ongoing difficulty and increasingly poor grades as an at-risk student. 
Both over- and undergeneralizations can be considered as maladaptive beliefs because they are inaccurate and could lead to disengagement from learning. Learningrelated struggles are not always private experiences, as they can often happen in the presence of peers within the classroom. Students who believe they struggle with all dimensions of a subject (overgeneralization) may attempt to avoid using those skills at all costs in the interest of avoiding public shame in front of their peers. In addition to stalling development due to lack of practice and use of skills, this type of avoidance behaviour makes it difficult for educators to notice specific difficulties that may not have been formally identified and therefore are only experienced privately by the student. Both types of generalizations can also be avoided with clear communication about the specific nature of LD among professionals who make identifications, educators who work directly with students, and the identified students themselves.

Reconceptualizing LD as a network of difficulties. Given the variation both between and within types of LD, thinking about LD as a network of difficulties, rather than as a series of types and subtypes, may be more adaptive for all stakeholders. Where types and subtypes may encourage thinking by multi-dimensional skillset or subject, considering LD as a network of difficulties could promote the examination of specific needs as experienced by students that can fall within a skillset or span across subjects.

To use reading as an example, this multi-dimensional skill can be visualized as a network of primary skills. To be a successful reader, one must learn to read words correctly and fluently, and eventually make sense of collections of words (sentences, paragraphs, articles). Each skill is important to effective reading, and therefore a deficit in one or more areas could result in difficulties with reading for the student experiencing them. $L D$ in reading can be identified based on a specific deficit in any one area related to reading (e.g., reading comprehension), or any combination of areas (APA, 2013).

In thinking about LD as a network of difficulties, the focus for each identified student is on the specific difficulty or difficulties experienced by the student, rather than on a subject (e.g., reading) that could lead to both types of generalizations. Additionally, thinking about LD as a network of difficulties suggests interconnections, specifically among difficulties and subjects. Specific cognitive abilities are necessary for success in a number of subjects.

A core deficit in shifting ability could be viewed as a common root of severe difficulties in reading fluency (LD in reading) and severe difficulties in mathematics computational speed (LD in mathematics) because of the focus on the pace of moving among information sources (Miyake et al., 2000). Similarly, a core deficit in planning, also considered an executive function behaviour by some researchers (Naglieri \& Goldstein, 2013), could be considered a common root of severe difficulties in reading unstructured text (LD in reading), solving multi-step problems in mathematics (LD in mathematics), and composition of writing (LD in writing) because of the focus on deciding on and implementing a course of action. With a focus on the specific cognitive deficit rather than on the subject, both educators and identified students can assess learning situations and tasks to anticipate difficulties that will likely arise as a result of the deficit. 
The multi-dimensional nature of many subjects requires a more specific and informed understanding of identified learning difficulties that fall within their area for all stakeholders. Viewing LD as a network of difficulties could encourage students with LD to seek the appropriate accommodations for their specific difficulties (e.g., graphic organizer for reading comprehension deficit) and avoid what could be unnecessary or inappropriate accommodations (e.g., reader for LD in reading). Additionally, this view serves educators in their purposeful design of tasks for students with varying learning needs and strengths. Most importantly, when students with LD conclude their formal education, they will enter the world with knowledge of their specific difficulties or deficits and make decisions about their career with this knowledge in mind.

\section{Mindset}

As a result of a history of academic challenges and of experiencing difficulties that their peers do not experience, it is plausible that many students with LD would adopt a view that the source of their problems is within themselves rather than within the learning environment. While internalizing problems may not, in itself, be a maladaptive belief, it can become one when it is coupled with a maladaptive view of learning and ability. Dweck (2006) identified that when individuals attribute their successes and failures to abilities rather than effort, they are less likely to view themselves as capable of growth and learning in areas they are not already successful in. One study reported that, along with lower expectations for academic success, students with LD were also more likely than their peers without LD to attribute failure to a lack of ability (Valås, 2001). When students place an emphasis on abilities as determinants of success, they may feel that there is no purpose in making any effort in multi-dimensional subjects in which they experience difficulties in as a result of their LD.

Aside from the attributions students make about their learning successes and failures, Dweck (2006) also identified that some students endorse performance goals, which are focused on the judgements of others, rather than learning goals, which are focused on personal growth and change. In identifying as a disabled individual, a student may be sensitive to anything that could make them stand out from their peers. Individuals with hidden exceptionalities may feel that they can hide their disability if they simply ignore more public or obvious support and do their best to avoid situations that create problems for them. When students with LD care more about fitting in and avoiding accommodations that make them stand out, academic challenges associated with LD will not cease to exist. Blackwell, Trzesniewski, \& Dweck (2007) found that the tendency for students to make ability attributions and to endorse performance goals contributes to a maladaptive mindset, given their relationship with poor academic achievement. Individuals with LD may believe that their exceptionality defines them and what they are not good at, rather than considering that the issues they experience may be in part a result of the learning environment.

Adapting teaching \& the International Classification of Functioning and Disability (ICF). For any students who have internalized the problems associated with LD and who possess a mindset with an emphasis on abilities as the source of success and difficulty, Dweck (2006) would suggest introducing a "growth mindset." Increasing 
research on neuroplasticity (Shaffer, 2016) makes it easier for educators to promote the idea that one's current difficulties and abilities are not necessarily indicative of what could be achieved with time and effort. Educators who endorse a growth mindset would value effort despite specific difficulties within and across multi-dimensional subjects, and would encourage students to continue to find ways to overcome obstacles and improve skills. Concern shifts from performance to personal growth; and more generally, individuals shift away from accepting exceptionalities as limitations and toward actively finding ways to learn and grown despite difficulties. Part of the difficulty for some students in shifting toward a more active and accountable mindset may be the internalization of problems associated with LD as something that is "wrong with them."

Medical models have largely been used in education to view and support students' learning needs (e.g., Cortiella \& Horowitz, 2014). Recently, however, there has been a shift from the medical models of disability to a functional model focusing on the problems of everyday life (Lillvist, 2010). The World Health Organization's (WHO) International Classification of Functioning Disability and Health (ICF; 2001, 2007) is more closely aligned with this functional model. ICF views disability and functioning "as outcomes [due to an interaction] between health conditions and contextual factors" (WHO, 2002, p. 10). The consequences of a particular disability, then, are context sensitive, as the environment both restrains and facilitates function (Lillvist, 2010; Simeonsson et al., 2006). The ICF aligns well with Stein's (2006) suggestion that the consequences of the disability need to be at the forefront of the conversation about intervention and treatment, since many "children with the same diagnoses ... have different types of consequences as a result of their disabling conditions" (p. 150).

Medical models provide accuracy in diagnosis and reflect cutting-edge empirical evidence of physical systems. Given the benefits that medical models of disability offer stakeholders, educators might consider the value of pairing a medical model with a functional approach within the classroom. In a period of development wrought with selfconfidence issues and emerging identity, it may be adaptive for students with LD to focus both on understanding their current abilities and specific difficulties as well as on how these interact with the ever-changing learning environment. Though medical models can guide the identification process, introducing a functional model within the classroom could pair well to combine accuracy of understanding with empowerment. When difficulties within the network have been identified for individual students, educators and students can think about the problems the learning environment could create given these difficulties and can make necessary changes in how learning is approached within the classroom.

ICF's focus on the functional aspects of an exceptionality, as well as the need to consistently re-evaluate functional needs based on the context of learning, can be seen in Corno's (2008) research. According to Corno (2008), "Good teachers capitalize on the capabilities and styles of their students, adjusting teaching for different conditions" ( $p$. 161). She called this process adaptive teaching. Adaptive teaching is nothing new; as both Corno (2008) and Snow (1982) noted, there are references to principles of adaptive teaching in Chinese, Hebrew, and Roman texts from as far back as the 1st century BCE. 
Adaptive teachers differentiate instruction guided by the principles of a Universal Design for Learning (e.g., Katz, 2012), sometimes unconsciously. Corno (2008) managed to summarize her research findings into a description of one lesson:

differentiation ... was not something [adaptive] teachers did routinely, or even an experience they could plan ahead for ... rather, their adaptions were spontaneously responsive to the individuals in their particular classrooms at the particular moments of instruction that occurred. These adaptions were informed by their own prior teaching history and experience with their curriculum. (p. 170)

Furthermore, adaptive teachers value diversity in students, and they embrace and nurture diverse characteristics. Adaptive teachers work with students to build relative weaknesses into strengths.

Adopting a functional approach informed by a medical model of disability for supporting students with LD in the classroom would place emphasis on considering changes that can be made to the learning environment. This approach would encourage educators to focus on adaptive design of the learning environment and effective communication with students with exceptionalities, and would encourage students with exceptionalities to focus understanding their specific challenges, assessing learning environments for anticipated difficulties, and advocating for appropriate supports.

\section{Significance of Maladaptive Beliefs}

Without the maladaptive behaviours that can result, generalizations and maladaptive mindsets would not necessarily be considered maladaptive beliefs. Just as beliefs promote action, they can also promote inaction. Learned helplessness can be understood as an increasingly passive pattern of behaviour, resulting from a pattern of thinking characterized by the belief that one is unable to change current circumstances as a result of past experiences (Seligman, 1995). Generalizations could lead to avoidance behaviour and disengagement from learning; and internalization of problems associated with LD, when paired with a maladaptive mindset, could also result in an increasingly passive pattern of learning behaviour. In the same study that found that students with LD had lower expectations than their non-LD peers, Valås (2001) also found that students who received special education support displayed more helpless learning behaviour than their low-achieving peers who did not receive support. Over time, the types of experiences students have in learning shape their perspectives and beliefs about their abilities as learners, and therefore shape the types of behaviours they engage in within learning environments.

\section{Toward an Alignment in Manifestation, Identification, Documentation, and Interpretation}

For beliefs about HIHE like LD to be adaptive, they should be accurate. A student whose interpretation of their exceptionality exactly matches its manifestations as learning difficulties is equipped to anticipate challenges rather than simply react to them. The alignment between how HIHE are interpreted and how they manifest as specific learning 
difficulties is therefore influenced by individual beliefs, but there are other areas related to the identification process that should also align with interpretation and manifestation.

Using LD as an example, there should be alignment among the way in which LDs manifest as learning difficulties for students, how they are interpreted by educators and by students themselves, how they are formally identified, and the types of supports suggested to address identified difficulties. As can be seen in Figure 1, we propose a framework that identifies each sector involved in the identification process, along with factors that influence the alignment of sectors. We argue that the alignment of sectors depends on the identified factors.

Figure 1. The MIDI Framework for Alignment of Sectors Involved in the Identification Process for High-Incidence Hidden Exceptionalities

\section{The MIDI Framework}

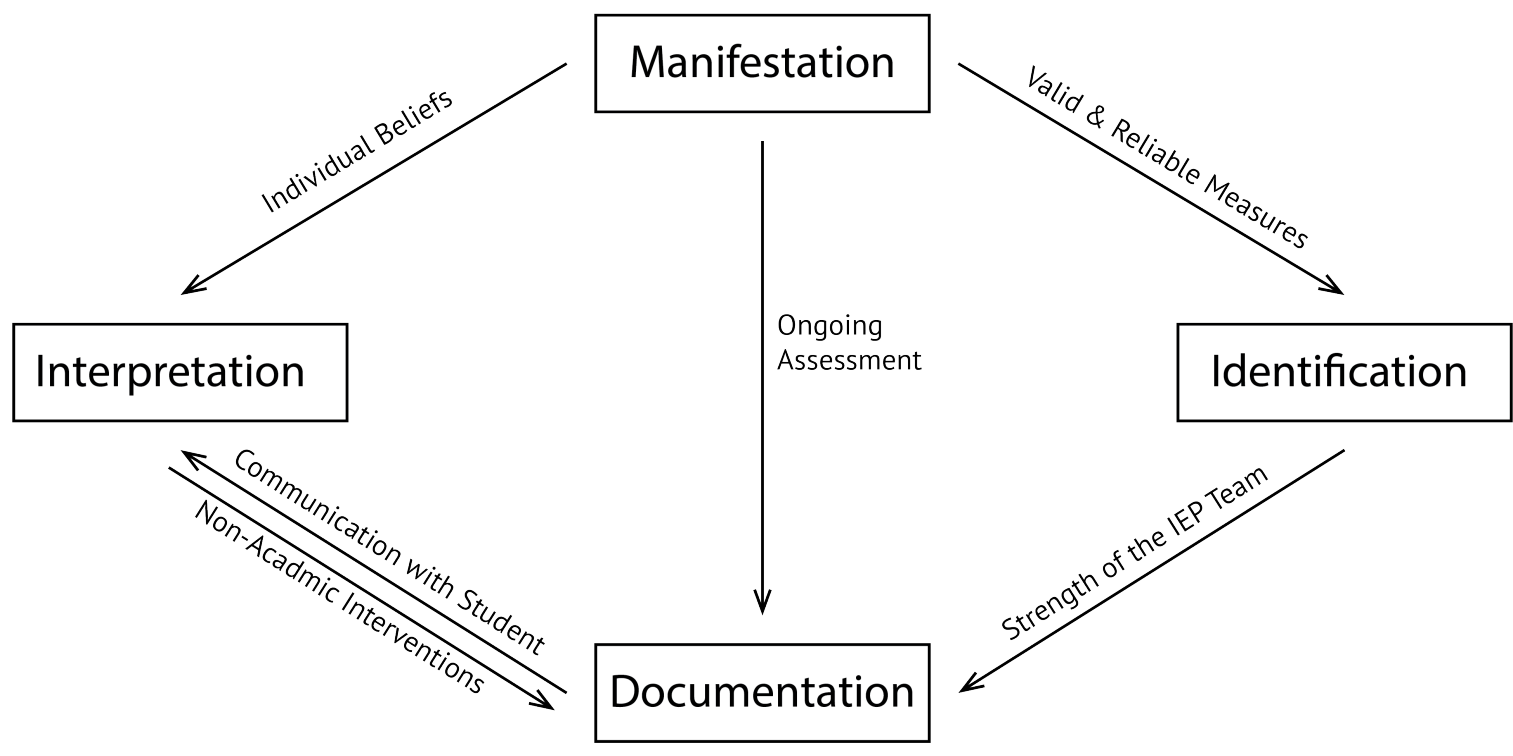

\section{Sectors}

The manifestation sector within the manifestation, identification, documentation, and interpretation (MIDI) framework is exactly how the exceptionality affects learning. For an individual with a specific LD in reading comprehension, it may be the experience of difficulty when integrating and connecting ideas in text, and finding the main idea. The identification sector is the exceptionality that is identified, including how specific or general the identification is (e.g., learning disability in reading vs. specific deficit in reading comprehension). The documentation sector comprises the changes that are to be put in place to support the identified difficulties. This refers to the document(s) used to identify exceptionalities and how they are supported in the classroom, such as the IEP. The interpretation sector, as presented in the MIDI framework, is focused on the student's understanding of their exceptionality in terms of how it affects their learning. The interpretation sector could also involve how educators and other stakeholders interpret exceptionalities given the influence this can have on students (e.g., Mouzourou, 2009); 
however, the identified factors in the MIDI framework are based on the interpretation of the student and what the student believes about the nature of their exceptionality.

\section{Factors}

Sectors within the MIDI framework are connected by factors that determine alignment; for two sectors to be aligned, they depend on the factor that connects them. As HIHEs manifest as learning-related difficulties, students who experience them form beliefs about them based on these experiences-beliefs that then influence how they interpret what their exceptionality means. Therefore, the alignment of manifestation and interpretation depends on individuals' beliefs about their experiences of learningrelated difficulties.

Individuals who make identifications do so with established measures that are considered both reliable and valid. This could be done by professionals who use psychometric assessments to assess for significant difficulties related to learning, or by educators through class assignments and other assessments, as well as observations and notes. If unreliable or invalid measures are used to make identifications, there could be misalignment between how HIHEs manifest as learning-related difficulties, and how they are specifically identified. The alignment of identification and manifestation depends on the validity and reliability of measures used to make identifications. Valid and reliable measures should not only identify the multi-dimensional skill(s) that relate to the experienced difficulties, but also specifically what challenges within the network of difficulties are experienced by the individual.

The alignment between how HIHEs manifest and how they are documented on individual student support plans depends on ongoing assessment. As identified students progress through school, the learning-related challenges they face as a result of their HIHE may change; and therefore a review of their experience should be made regularly. In Ontario, the team tasked with creating and maintaining the IEPs are required legally to meet "at least once in each school year" (OME, 2017, p. D2) to review the support plan, including a consideration of how things have changed for the student, and whether the supports detailed on the plan remain appropriate. The alignment of documentation and manifestation depends on ongoing assessment of identified students' needs.

The alignment between identification and documentation depends on the strength of the support team that builds the support plan (e.g., IEP) for students. A strong team would consider the identification, student, environment, and other relevant factors and recommend supports that address the identification (OME, 2017). When an assessment is made by a professional outside of the school, the identification is given to the support team, whose role becomes how to match the identification with appropriate supports. When the team is also responsible for making the identification, they rely on using artifacts of learning from the student to inform the types of supports that could be used to overcome learning-related difficulties going forward. The strength of this team in considering options and appropriately matching identifications (i.e., that they make or that are made for them) with supports determines how well identification and documentation align. 
The alignment of interpretation and documentation depends on how well the support plan is communicated to the student by members of the support team. In some cases, the student could be a part of the team that determines what goes into the support plan, and in other cases the student may not even be aware that they have a plan. How well a students' interpretation of their HIHE and the documentation of the plan to support them align depends on what information is communicated to the student, and how it is communicated to them.

The MIDI framework isolates individual sectors related to the identification process, and identifies factors that can influence the alignment among sectors. The framework provides direction for examining the effectiveness of the identification process for students with HIHE.

\section{Concluding Thoughts}

In this paper we aimed to shed light on the importance of considering students' beliefs about HIHE, using LD as an example. We argue that educators, as a factor within the MIDI framework that determines the alignment between manifestation and interpretation, can focus on reconceptualizing the way they think (and talk) about LD, as well as taking a functional approach informed by a medical model to support students with LD. Both of these "adaptive responses" represent non-academic supports in that they do not focus on skill deficits, but rather on individual beliefs about deficits. The proposed responses also overlap with the factor focused on communication with the student, which determines the alignment of interpretation with documentation; we suggest that the way educators think about HIHE can inform the way they talk to students about them.

Interventions that focus on supporting identified skill deficits are important, but we argue for an additional focus on what could be considered "secondary challenges," like maladaptive beliefs, that form because of the primary skill-related challenges. The MIDI framework provides a picture of what could be considered secondary challenges, where poor execution of any of the sectors in the identification process, excluding individual beliefs, could lead to a misalignment in factors related to the identification process. In the proposed framework, we imply the importance of consideration of all factors and sectors by the support team, as well as attention to how the student understands the process and their own learning-related challenges. Despite a dearth of research on the validity and reliability of measures for assessing learning and learning-related challenges (e.g., Ticha, Espin, \& Wayman, 2009; Yopp, 1988), the framework highlights areas of focus for future research both for researchers and educators - to determine what processes could be improved and whether or not factors are aligning in each case. Both the emphasis on students' beliefs about HIHE and the presentation of the MIDI framework represent our efforts to emphasize the importance of the student perspective in the identification process. Most importantly, the changes we suggest to promote adaptive beliefs about HIHE and the considerations we lay out with the sectors in the MIDI framework do not necessitate redesigning structures already in place regarding the identification process. 


\section{References}

American Psychiatric Association. (2013). Diagnostic and statistical manual of mental disorders (5th ed.). Arlington, VA: Author.

Baird, G. L., Scott, W. D., Dearing, E., \& Hamill , S. K. (2009). Cognitive self-regulation in youth with and without learning disabilities: Academic self-efficacy, theories of intelligence, learning vs. performance goal preferences, and effort attributions. Journal of Social and Clinical Psychology, 28, 881-908.

Blackwell, L. S., Trzesniewski, K. H., \& Dweck, C. S. (2007). Implicit theories of intelligence predict achievement across an adolescent transition: A longitudinal study and an intervention. Child Development, 78, 246-263. doi:0009-3920/2007/7801-0014

Buttner, G., \& Hasselhorn, M. (2011). Learning disabilities: Debates on definitions, causes, subtypes, and responses. International Journal of Disability, Development and Education, 58, 75-87.

Corno, L. (2008) On teaching adaptively. Educational Psychologist, 43, 161-173, doi:10.1080/00461520802178466

Cortiella, C., \& Horowitz, S. H. (2014). The state of learning disabilities: Facts, trends, and emerging issues (3rd ed.). New York, NY: National Center for Learning Disabilities.

DeLuca, C., Hutchinson, N. L., deLugt, J. S., Beyer, W., Thornton, A., Versnel, J., ... Munby, H. (2010). Learning in the workplace: Fostering resilience in disengaged youth. Work: A Journal of Prevention, Assessment, and Rehabilitation, 36, 305-319. doi:10.3233/WOR-2010-1032

Dweck, C. S. (2006). Mindset: The new psychology of success. New York, NY: Random House.

Geary, D. C. (2006). Learning disabilities in arithmetic: Problem-solving differences and cognitive deficits. In H. L. Swanson, K. R. Harris, \& S. Graham (Eds.), Handbook of learning disabilities (pp. 199-212). New York, NY: Guilford Press.

Finlay, B. (2011). Categories of exceptionalities (Memorandum). Retrieved from the website of the Ontario Ministry of Education, http://www.edu.gov.on.ca/eng/general/elemsec/speced /2011categoryexception.pdf

Gage, N. A., Lierheimer, K. S., \& Goran, L. G. (2012). Characteristics of students with high-incidence disabilities broadly defined. Journal of Disability Policy Studies, 23(3), 168-178. doi: $10.1177 / 1044207311425385$

Gargiulo, R. M. (2012). Special education in contemporary society. Los Angeles, CA: Sage.

Gibbs, S., \& Elliott, J. (2015). The differential effects of labeling: How do 'dyslexia' and 'reading difficulties' affect teachers' beliefs. European Journal of Special Needs Education, 30, 323337. doi:10.1080/08856257.2015.1022999

Hutchinson, N. L. (2017). Inclusion of exceptional learners in Canadian schools: A practical handbook for teachers (5th ed.). Toronto, ON: Pearson Education Canada.

Kane, M. J., \& Engle, R. W. (2002). The role of prefrontal cortex in working-memory capacity, executive attention and general fluid intelligence: An individual differences perspective. Psychonomic Bulletin \& Review, 4, 637-671.

Katz, J. (2012). Teaching to diversity: The three-block model of Universal Design for Learning. Winnipeg, MB: Portage \& Main Press.

Klassen, R. N. (2010). Confidence to manage learning: The self-efficacy for self-regulated learning of adolescents with learning disabilities. Learning Disabilities Quarterly, 33, 19-30.

Lackaye, T., Margalit, M., Ziv, O., \& Ziman, T. (2006). Comparisons of self-efficacy, mood, effort, and hope between students with learning disabilities and their non-LD-matched peers. 
Learning Disabilities Research \& Practice, 21, 111-121. doi:10.1111/j.15405826.2006.00211.x

Learning Disabilities Association of Ontario. (2018). What are LDs? [Web page]. Retrieved from http://www.ldao.ca/introduction-to-ldsadhd/what-are-lds/

Lillvist, A. (2010). Observations of social competence of children in need of special support based on traditional disability categories versus a functional approach. Early Child Development and Care, 180, 1129-1142, doi:10.1080/03004430902830297

MacMaster, K., Donovan, L. A., \& MacIntyre, P. D. (2002). The effects of being diagnosed with a learning disability on children's self-esteem. Child Study Journal, 32, 101-108.

Miyake, A., Friedman, N. P., Emerson, M. J., Witzki, A. H., Howerter, A., \& Wager, T. D. (2000). The unity and diversity of executive functions and their contributions to complex "frontal lobe" tasks: A latent variable analysis. Cognitive Psychology, 41, 49-100. doi:10.1006/cogp.1999.0734

Mouzourou, C. (2009). Same but different: Exploring young children's understandings about disability (Doctoral dissertation). University of Illinois at Urbana-Champaign, IL. Retrieved from Proquest Dissertations and Theses (UMI No. 3392230), www.proquest.com

Naglieri, J., \& Goldstein, S. (2013). Comprehensive executive functioning inventory technical manual. Toronto, ON: Multi-Health Systems.

Ontario Ministry of Education. (2017). Special education in Ontario: Kindergarten to Grade 12. Toronto, ON: Queen's Printer for Ontario.

Osterholm, K., Nash, W. R., \& Kritsonis, W. A. (2007). Effects of labeling students "learning disabled": Emergent themes in the research literature 1970 through 2000. Focus on Colleges, Universities, and Schools, 1, 1-11.

Shaffer, J. (2016). Neuroplasticity and clinical practice: Building brain power for health. Frontiers in Psychology, 7(1118). doi:10.3389/fpsyg.2016.01118

Seligman, M. E. P. (1995). The optimistic child. Sydney, NSW: Random House Australia.

Simeonsson, R. J. (2009). ICF-CY: A universal tool for documentation of disability. Journal of Policy and Practice in Intellectual Disabilities, 6, 70-72. doi:10.1111/j.1741-1130.2009.00215.x

Simeonsson, R. J., Scarborough, A. A., \& Hebbeler, K. M. (2006). ICF and ICD codes provide a standard language of disability in young children. Journal of Clinical Epidemiology, 59(4), $365-373$.

Snow, R. E. (1982). Education and intelligence. In R. J. Sternberg (Ed.), Handbook of human intelligence (pp. 493-596). London, UK: Cambridge University Press.

Stein, M. (2006). Research review: Young people leaving care. Child \& Family Social Work, 11, 273-279.

Ticha R., Espin C., \& Wayman, M. W. (2009). Reading progress monitoring for secondary-school students: Reliability, validity, and sensitivity to growth of reading aloud and maze selection measures. Learning Disabilities Research \& Practice, 24, 132-142.

Valås, H. (2001). Learned helplessness and psychological adjustment: Effects of age, gender and academic achievement. Scandinavian Journal of Educational Research, 45, 71-90, doi:10.1080/00313830020042689

World Health Organization. (2001). International classification of functioning, disability and health. Geneva, Switzerland: Author.

World Health Organization. (2002). Towards a common language for functioning, disability and health. Retrieved from www.who.int/classifications/icf/training/icfbeginnersguide.pdf 
World Health Organization. (2007). International classification of functioning, disability and health (Children \& youth version). Geneva, Switzerland: Author.

Yopp, H. K. (1988). The validity and reliability of phonemic awareness tests. Reading Research Quarterly, 23, 159-177. doi:10.2307/747800

\section{Authors' Note}

Correspondence concerning this article should be addressed to Ian A. Matheson, Queen's University, 511 Union Street, Kingston, ON, K7M 5R7, Canada. Email: ian.matheson@queensu.ca 